\title{
Design and Implementation of Transfusion Auxiliary Device by Patient Manual Control Dressing Change
}

\author{
Jiang Jin-gang ${ }^{1,2} *$, Shen Rui-chao ${ }^{2}$, Wang Bao-fu ${ }^{2}$, Gu Bo-yang ${ }^{3}$, \\ Tang Hai-bo ${ }^{2}$ and Jiang Ze-hao ${ }^{2}$ \\ ${ }^{1}$ Robotics \& its Engineering Research Center, Harbin University of Science and \\ Technology, Harbin 150080, China \\ ${ }^{2}$ Faculty of Vehicle Engineering and Mechanics, Dalian University of Technology, \\ Dalian 116024 China \\ ${ }^{3}$ School of Mechanical and Power Engineering, Harbin University of Science and \\ Technology, Harbin 150080 China \\ *jiangjingang@hrbust.edu.cn
}

\begin{abstract}
Transfusion auxiliary device by patient manual control dressing is proposed. It is realized by one-way rotation ratchet mechanism, which is consistent of slider-crank mechanism and double pawl ratchet mechanism. Dressing change is automatically realized by patient manual control rope. This reduces the labor intensity of the medical staff, shortens the waiting time of the patients. This device has the advantage of low cost, large market space.
\end{abstract}

Keywords: Transfusion auxiliary device, Patients manual control dressing change, Ratchet mechanism

\section{Introduction}

China has become transfusion great power by means of Chinese 8 bottles per capita transfusion. Public data shows that China's transfusion market capacity is more than 10 billion bottles (bags). It is equivalent to 8 bottles per people for 13 billion people. It is higher than 2.5-3.3 per bottle of international level [1]. By the end of 2013, the total number of registered nurses is 2.783 million in China [2]. Even if all nurses take part in transfusion services, every nurses will give patients to transfusion more than 4000 bottles average every year. The labor intensity of medical staff is large. In 2012, Chinese community doctors release a nursing questionnaire survey, and the results show that nearly ninety percent of the nurse said the labor intensity is high or higher [3]. The labor intensity, mental pressure of nurses is high, and the labor cost and income is out of proportion.

In the intravenous drip room or ward of the hospital, a lot of patients usually transfusion simultaneously. After a bag of liquid medicine is transfusion completely, the operation of dressing change is needed. And the work is often performed by one or a few nurses. Because of a lot of patients, a few patients at different wards often dressing change simultaneously. Medical staff need to carry liquid medicine to run among different wards. So this condition add the labor intensity. In the process of dressing change, the patients need to shut down transfusion device, and wait for dressing change. This will affect some patient's mood and enable it irritable. How to make patients to do simple operation to achieve dressing change? This is in favour of reducing the workload of medical staff, improving the patient's mood. It is a need to solve the problem.

In view of the above problem, the existing vein transfusion device is analyzed. Transfusion auxiliary device by patient manual control dressing change is designed. 
Mechanical design without electrical control is adopted. It also make patients to control the switch of different bottle. This mode enable the medical staff an installation to realize the automatic multi dressing change repeatedly. And this can improve the work efficiency. To a certain extent, this alleviates the present situation of medical resources in our country. The production cost of the design is relatively low, simple maintenance, easy for product promotion.

\section{Analysis on Existing vein Transfusion Device}

\subsection{Analysis on Traditional vein Transfusion Device}

Existing transfusion method in hospital is manually change medicine by the patients call to medical personnel on time. The infusion tube is pulled out from an empty bottle in the operation of dressing change. Then insert the infusion tube and exhaust pipe to the new bottle. The operation of dressing change is achieved after fixed vent and opening the switch of infusion tube. This operation is as shown in Figure 1.

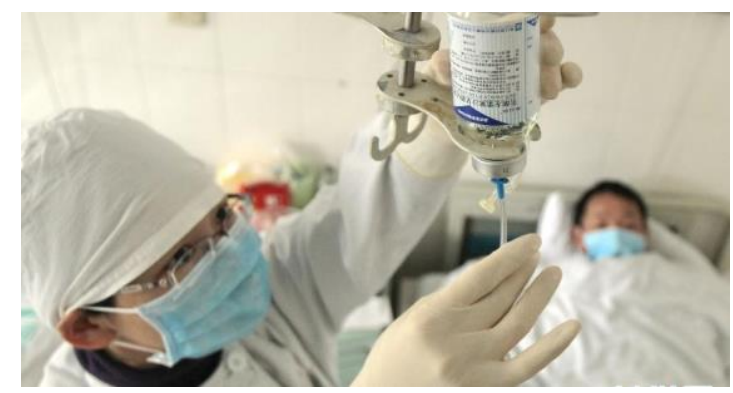

\section{Figure 1. The Existing Dressing Change Method of Infusion Apparatus}

\subsection{Analysis on Automatic Transfusion Pump}

The work principle of automatic transfusion pump is, transfusion pump is controlled by electronic. It can display the real-time monitoring status of the transfusion process, ensure the drug dosage, input the fluid velocity precisely, select transfusion tube automatically, and switch the input liquid medicine. Automatic transfusion pump is as shown in Figure 2.

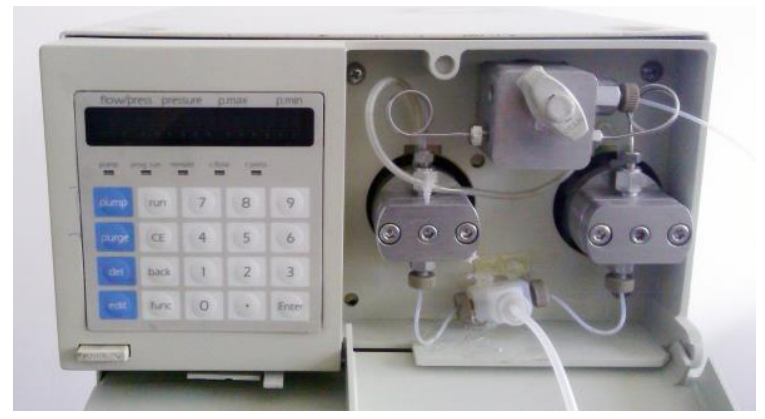

Figure 2. Automatic Transfusion Pump

The automatic selection of transfusion tube is to switch the input liquid medicine. The work principle is to enable the different transfusion tube collect multi-ported valve. One end of the valve connect needle, and the order of input liquid medicine is controlled by the electronic [4-5].

Automatic transfusion pump in use function is perfect. Its comprehensive performance is superior. And this reduces the labor intensity of medical staff greatly. However, the price of transfusion tube is expensive, and it is not suitable for large-scale promotion use. 
The price of domestic transfusion tube is between RMB 3000 yuan and 15000 yuan commonly.

In this paper, combined with the above problems, through the comparison and analysis of the transfusion type of the existing transfusion device, and considering the truncation of different transfusion tube, the different transfusion tube is assembled into a transfer device. The order of input liquid medicine is controlled by through the way of manual control.

The whole idea is based on a kind of unidirectional rotation ratchet mechanism. The mechanism is as shown in Figure 3, consistent of the slider-crank mechanism and double pawl ratchet mechanism [6].

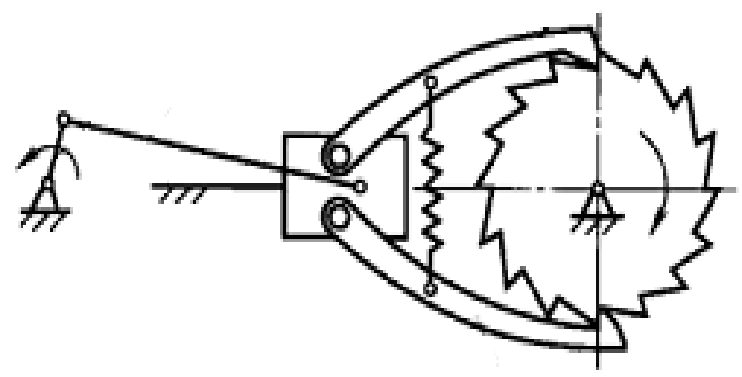

Figure 3. Unidirectional Rotation Ratchet Mechanism

\section{Structure and Function of Transfusion Auxiliary Device}

Transfusion auxiliary device by patient manual control dressing change is consistent of operative part, transmission part and control part. Operative part is composed of funnel for liquid storage with multi-diameter shaft, ratchet and pawl for dressing change, tension spring and perfusion tube fixed plate. Transmission mechanism is composed of the slider, sliding rail, reset spring, fixed pulley and support frame. Control end is a rope controlled by hand.

The structure of transfusion auxiliary device by patient manual control dressing change is as shown in Figure 4 and Figure 5. In the operative part, the diameter of the upper edge of funnel for liquid storage with multi-diameter shaft is $34 \mathrm{~mm}$, the major diameter and minor diameter of center ladder support shaft are $12 \mathrm{~mm}$ and $8 \mathrm{~mm}$ respectively. The above adder shaft has the thread. The ladder shaft has the size of $3 \mathrm{~mm}$ hole. It is embedded the base. The hole is connected with the outside. In the process of the work, liquid medicine is come together to here. And it connect the overall tube and needle through the hole to intravenous drip for patients. The structure of dressing change is composed of ratchet, pawl and perfusion tube fixed plate. The number of teeth of ratchet for dressing change is 16 , the module of gear is 3 , and the thickness is $6 \mathrm{~mm}$. A circular groove is on the ratchet, and the major diameter and minor diameter of the ratchet is $34 \mathrm{~mm}$ and $30 \mathrm{~mm}$ respectively. The groove depth is $4 \mathrm{~mm}$. The diameter of hole is $3 \mathrm{~mm}$, and it is located at $16 \mathrm{~mm}$ from the axis of ratchet wheel. The hole diameter of ratchet center shaft is $8 \mathrm{~mm}$, and the ratchet installed on the ladder shaft with the funnel for liquid storage. They can be relatively rotation. Perfusion tube fixed plate located at the ratchet connect with the ladder shaft with the funnel for liquid storage by screw joint. Circular convex plate is located perfusion tube fixed plate. The major diameter and minor diameter of the circular convex plate is $34 \mathrm{~mm}$ and $30 \mathrm{~mm}$ respectively. And the height is $4 \mathrm{~mm}$. The diameter of 16 holes is $3-4 \mathrm{~mm}$, and it is located at $16 \mathrm{~mm}$ from the center of perfusion tube fixed plate. It is used with the ratchet for dressing change cooperatively. Perfusion tube fixed plate connects with the funnel for liquid storage, and they is relatively rest. The ratchet for dressing change moves relatively with them. When the hole of the ratchet for dressing change is coincide with the hole of the perfusion tube fixed plate, liquid medicine flows 
into the funnel for liquid storage. In working, transfusion tube connected with different transfusion bottle insert into different holes of perfusion tube fixed plate. Patient pull the pawl for dressing change by manual control, and drive the rotation of the ratchet for dressing change in order to control the transfusion of different transfusion tube. So the order of transfusion is controlled manually by the change of transfusion bottle or bag.

1 base, 2 funnel for liquid storage with multi-diameter shaft, 3 ratchet and pawl for dressing change, 4 perfusion tube fixed plate, 5, 6 pawl for dressing change, 7 tension spring, 8, 9 fixed pulley and its support frame, 10 sliding rail, 11 slider, 12 reset spring, 13 a rope controlled by hand, 14 hole for rope, 15 the overall tube, 16 transfusion tube.

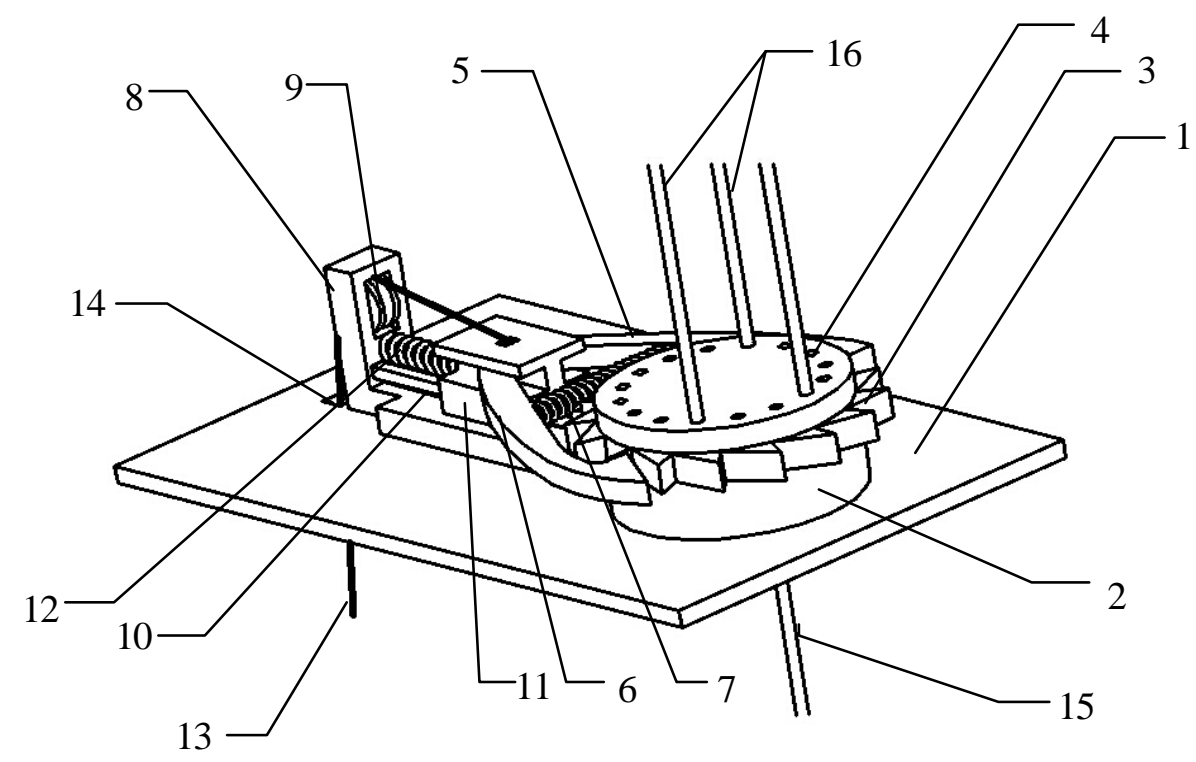

Figure 4. Structure of Transfusion Auxiliary Device by Patient Manual
Control Dressing Change

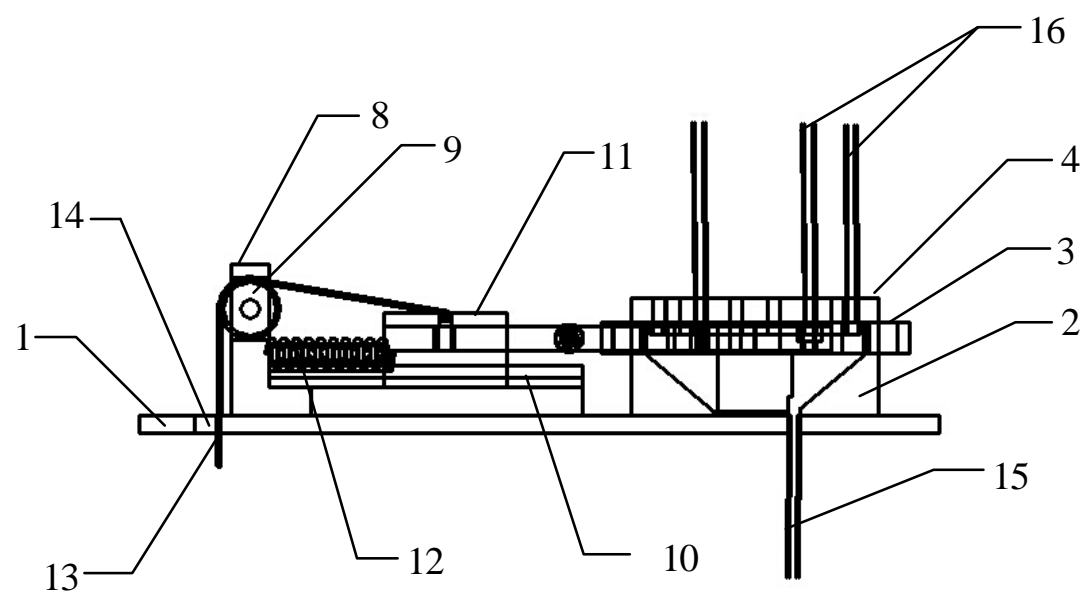
Figure 5. Front View of Transfusion Auxiliary Device by Patient Manual
Control Dressing Change

For control end and transmission part, the slider rail connects with the base. And they locate at the left of the funnel for liquid storage. Fixed pulley and its support frame locates at the left of the slider rail. The two sides of the slider rail connect with pawl for dressing change, and the other end connects with reset spring. Control end is a rope controlled by 
hand. The other end of rope connect with driving block by the fixed pulley. In service, patient pull the rope, and drive the movement of the slider block along with slider rail towards left movement. And then the pawl is pulled, drive the rotation of the ratchet for dressing change. So the order of transfusion is controlled manually by the change of transfusion bottle or bag.

Three-dimensional model of transfusion auxiliary device by patient manual control dressing change is as shown in Figure 6.

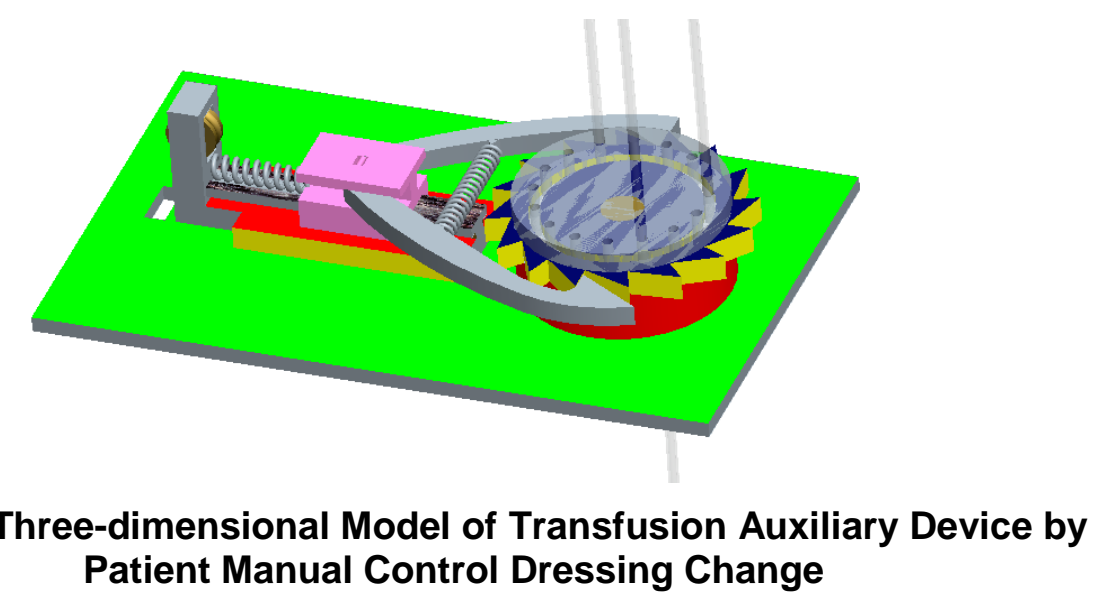

\section{Material Selection of Operative Device}

On the basis of the analysis of the structure and function, in order to achieve the function of dressing change by the manual control, the key is to solve the problem of leakproofness of operative device and the relative movement of each component of operative device. It should ensure the transfusion no leakage, and reduce the friction between the ratchet for dressing change and perfusion tube fixed plate as much as possible. For this reason, in order to meet these requirements, special attention should be paid to the selection on the material of the ratchet for dressing change and perfusion tube fixed plate.

Aiming at the problem, this type of material problem existing in the engineering is compared by the analogy method. Through the analysis on the existing problem of motive seal, it is similar with the problem of valve seal. Direct contact type and rotation type motive seal of the moving part and the stationary part is adopted. The stuffing box type is the gland nut type. The feature of this type is small boundary dimension, restricted force. And it is suitable for small diameter seal [7]. Transfusion auxiliary device by patient manual control dressing change designed in this paper is used in non-tax environment. And it has not no high requirements on speed. So the selection of the padding is rubber Oring. And its feature is good sealing, corrosion resistance and low friction coefficient [8]. Furthermore, the hole located at the ratchet for dressing change and perfusion tube fixed plate also adopts rubber O-ring to realize the sealing. And it can reduce the liquid leakage of the joint between transfusion tube and perfusion tube fixed plate, and between funnel for dressing change and the transfusion tube.

\section{Force Calculation of the Rope by Manual Control}

Through the functional analysis of transfusion auxiliary device by patient manual control dressing change, when the change of transfusion bottle or bag is realized by manual control, the patients need to pull the manual control rope. And it should overcome the spring contraction force generated by the compression of reset spring. At the same time, when pulling the slider, the movement of the pawl drive the ratchet rotation. In this process, it should overcome the friction between the ratchet for dressing change and the 
perfusion tube fixed plate. Here supposed there is no friction between slide block and the slide rail. That is

$$
F=F_{K}^{\prime}+F_{f}^{\prime}
$$

Where, $F$ is the tensile force of manual control end, $F_{K}^{\prime}$ is the projection of contraction force $F_{K}$ of reset spring along with manual control rope direction, $F_{K}$ is the projection of the friction force $F_{f}$ between between the ratchet for dressing change and the perfusion tube fixed plate along with manual control rope direction. Force analysis of manual control rope end is as shown in Figure 7.

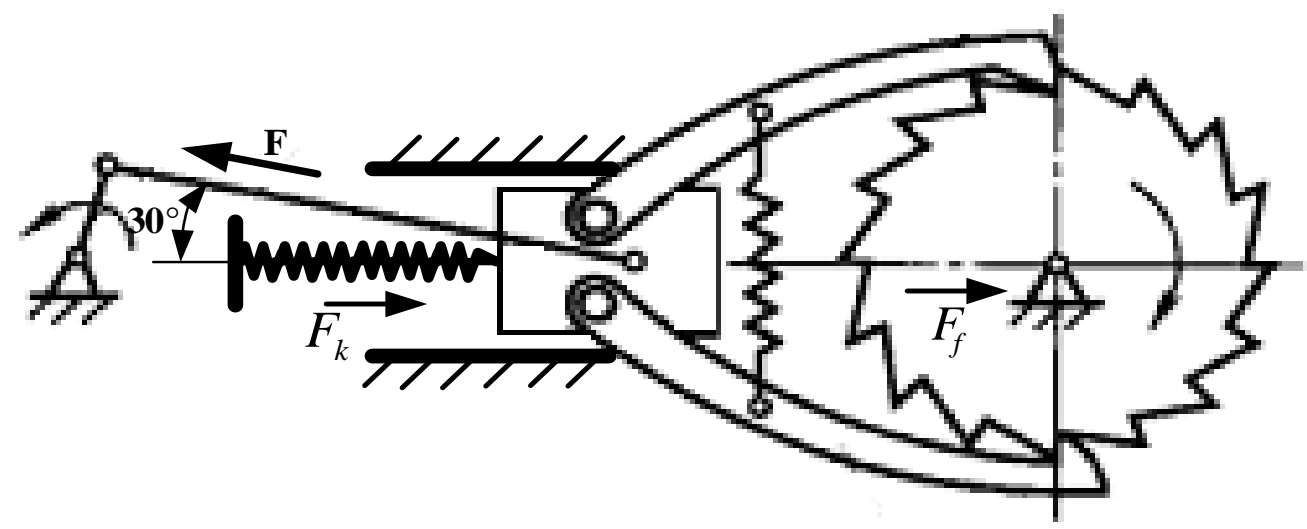

Figure 7. Force Analysis of Manual Control Rope End

Mechanical Model of Manual Rope End is as Shown in Figure 8.

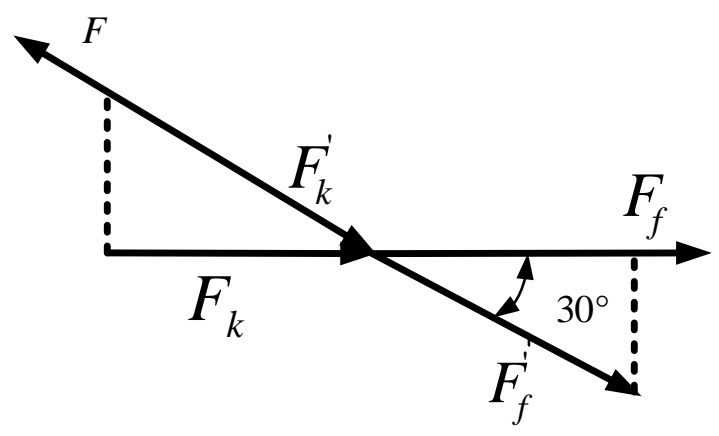

Figure 8. Mechanical Model of Manual Rope End

Where,

$$
F_{K}=k \cdot x
$$

Where, $k$ is the stiffness coefficient of the spring, $x$ is spring compressed length, and $k=3 \mathrm{~N} / \mathrm{m}$. Because the number of teeth of ratchet for dressing change is 16 , the module of gear is 3 . So the radius is $24 \mathrm{~mm}$, the perimeter is $150.72 \mathrm{~mm}$. When pulling the slider block, the displacement of the ratchet for dressing change is less than $10 \mathrm{~mm}$, here take $x=10 \mathrm{~mm}=0.01 \mathrm{~m}$.

$$
\begin{gathered}
F_{K}=k \cdot x=3 \times 0.01=0.03 \mathrm{~N} \\
F_{f}=\mu \cdot F_{N}
\end{gathered}
$$


Where, $\mu$ is the friction coefficient between the ratchet for dressing change and the perfusion tube fixed plate, and $\mu=0.12$. Because the ratchet for dressing change is at rest relative to the perfusion tube fixed plate. So the friction can be seen as a static friction with no relative rotation and liquid friction. And then the size of friction is proportional to positive pressure. The pre-tightening force of the perfusion tube fixed plate is $0.06 \mathrm{Mpa}$. So,

$$
\begin{gathered}
F_{f}=\mu \cdot F_{N}=0.12 \times 0.06 \times 10^{6} \times 3.14 \times 0.024^{2} \approx 13.2 \mathrm{~N} \\
F=F_{K}^{\prime}+F_{f}^{\prime}=\left(F_{K}+F_{f}\right) / \cos 30^{\circ} \approx 15 \mathrm{~N}
\end{gathered}
$$

Based on above analysis, when the change of transfusion bottle or bag is realized by manual control, the tensile force of manual control rope pulled by the patient is $15 \mathrm{~N}$, it is suitable for most patients.

The physical model of transfusion auxiliary device by patient manual control dressing change is as shown in Figure 9 and Figure 10.

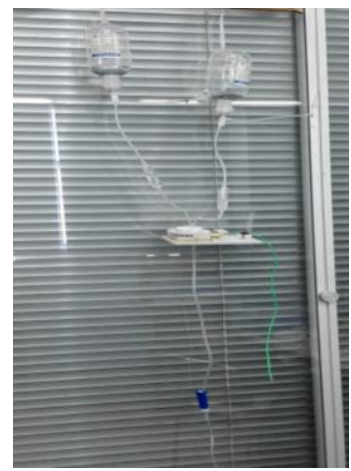

Figure 9. The Physical Model

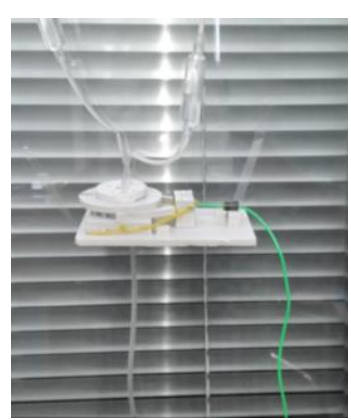

Figure 10. Topography of the Physical Model

\section{Conclusions}

For reducing the workload of doctors, Transfusion auxiliary device by patient manual control dressing is proposed on the basis of the analysis on the existing transfusion device. It is realized by one-way rotation ratchet mechanism, which is consistent of slider-crank mechanism and double pawl ratchet mechanism. Material of operative device is selected considering the problem of leak-proofness of operative device and the relative movement of each component of operative device. Force of the rope by manual control is calculated, and it is suitable for most patients. The physical model of transfusion auxiliary device by patient manual control dressing change is established. The device reduces the labor intensity of the medical staff, shortens the waiting time of the patients.

\section{Acknowledgements}

We are extremely grateful to the anonymous referees for their most insightful and constructive comments, which have enabled us to improve the manuscript significantly. The authors are also grateful to the Editor in Chief and the technical editor for reviewing and editing the paper. This research was supported by the Undergraduate Innovative Experiment Project of Harbin University of Science and Technology. 
International Journal of $u-$ and $\mathrm{e}-$ Service, Science and Technology

Vol.8, No. 11 (2015)

\section{References}

[1] L. Peng, "Transfusion power and transfusion profit", Shanghai Enterprise, vol. 10, no. 18, (2013).

[2] C. De-mei, W. Qiao-qin, "Analysis on satisfaction and influencing factors of transfusion outpatients and strategy", Journal of Nursing, (2010), vol. 17, no. 7, pp. 16-18.

[3] C. Tong, "Working principle and quality control of hospital transfusion pump", Metrology and Measurement Technique, (2012), vol. 39, no. 5, pp. 16-17.

[4] B. Jun-cheng, C. Mian-kang, Z. Na, J. Hai-xia, "Practice of medical infusion pump quality control", China Medical Devices, (2014), vol. 29, no. 5, pp. 95-97.

[5] O. Aardal, Y. Paichard, S. Brovoll, T. Berger, T. S. Lande, "Physical working principles of medical radar", IEEE Transactions on Biomedical Engineering, vol. 60, no. 4, (2013), pp. 1142-1149.

[6] S. Kai-yuan, "Common mechanism design and application graphic illustration", Beijing: Chemical Industry Press, (2010).

[7] L.Chuan-jun, M. Man, W. Hong-rui, Z. Yi, X. Guang, "Typical structures, materials and characteristics of dynamic seals for valves", Chinese Hydraulics and Pneumatics, vol. 5, (2014), pp. 118-121.

[8] J. Jin-gang, X. Xiao-lei, W. Zhao, L. Yun-feng, C. Shi-jia, S. Jing-hao, "Optimal structure design of multi-functional folding drawing board based on TRIZ theory", International Journal of Hybrid Information Technology, (2015), vol 8, no. 2, pp. 397-408.

[9] F. Li-jun, Z. Xi-bin, "Selection and design of O-ring rubber seal", Modern Vehicle Power, (2008), no. 3, pp. 46-48. 